\title{
Current-induced vortex dynamics and pinning potentials probed by homodyne detection
}

\author{
J.-S. Kim, O. Boulle, S. Verstoep, L. Heyne, J. Rhensius, ${ }^{*}$ and M. Kläui ${ }^{\dagger}$ \\ Fachbereich Physik, Universität Konstanz, Universitätsstr. 10, D-78457 Konstanz, Germany \\ L. J. Heyderman \\ Laboratory for Micro- and Nanotechnology, Paul Scherrer Institute, 5232, Villigen PSI, Switzerland \\ F. Kronast \\ Helmholtz-Zentrum Berlin für Materialien und Energie GmbH, Berlin, Germany \\ R. Mattheis \\ Institut für Photonische Technologien e.V., Jena, Germany \\ C. Ulysse and G. Faini \\ Phynano Team, Laboratoire de Photonique et de Nanostructures, CNRS, Marcoussis, France
}

(Received 22 June 2010; published 24 September 2010)

\begin{abstract}
Using a homodyne detection scheme, we show that we can determine the polarity and chirality of a magnetic vortex in an asymmetric magnetic disk as well as the resonance frequency and phase shift of the dynamic vortex gyration excited by a spin-polarized current. From systematic phase measurements, we deduce the relative contributions of the spin torque and the Oersted field, which is found to dominate the excitation. Local pinning sites in the disk lead to an increased resonance frequency and a reduced amplitude. This allows us to draw a map of the pinning sites and thus to characterize the full potential in the disk.
\end{abstract}

DOI: $10.1103 /$ PhysRevB.82.104427

PACS number(s): 72.25.Ba, 75.60.Ch, 75.75.-c

The dynamics of magnetic micron-sized elements with a vortex configuration is currently attracting considerable attention. ${ }^{1-8}$ This spin structure is characterized by an inplane magnetization curling around a small $(\sim 10 \mathrm{~nm}$ diameter) out-of-plane vortex core (VC). ${ }^{9}$ It has four energetically degenerate equilibrium states which are defined by the chirality (the clockwise or counterclockwise rotation sense of the in-plane magnetization) and the polarity (up or down direction of the VC). The lowest-order dynamical eigenmode of the vortex structure is a translational gyrotropic mode of the $\mathrm{VC}{ }^{10,11}$ It was recently shown that this mode can be resonantly excited using a small subgigahertz ac in-plane magnetic field ${ }^{2,3}$ or by the injection of an ac in-plane spinpolarized current ${ }^{4}$ and at resonance, the VC gyrates on a circular or elliptical orbit. ${ }^{2,5,7}$ In addition to displacing a VC, pulsed or ac magnetic fields or currents can also switch the VC polarity from up to down or vice versa ${ }^{5,6}$ which opens a new way to manipulate such a magnetic bit in a magnetic memory where the information is stored in the vortex polarity. ${ }^{12}$ Although the VC dynamics induced by an external magnetic field is now well understood, the exact nature of the force exerted on the VC when excited by an in-plane spin-polarized current has not been clearly identified. Whereas first experiments were interpreted in terms of the spin-transer effect only, ${ }^{4,6}$ it was claimed later that the current's Oersted field strongly contributes to the force on the VC. ${ }^{7}$ More recent experiments, on the contrary, determined the effect of the Oersted field on the VC dynamics to be negligible. ${ }^{13}$ In these previous experiments, ${ }^{7,13}$ the $\mathrm{VC}$ dynamics was characterized using time-resolved magnetic imaging techniques that prevented systematic measurements.

In this paper, we use versatile transport measurements employing the homodyne detection scheme ${ }^{14,15}$ to separate the different forces exerted on the $\mathrm{VC}$ and thus identify the relative contribution of the spin torque and the Oersted field on the VC dynamics. Homodyne detection is a powerful tool to characterize the dynamics of the vortex structure in a magnetic disk since it is very sensitive to the phase shift between the excitation and the VC motion, which contains the information on the acting forces. We show that the homodyne signal can further be used to identify the full magnetic state of the vortex structure (chirality and polarity) using a specific geometry. Our measurements reveal furthermore that the dynamics of a VC driven by an ac current can be strongly affected by local pinning sites. Using static external magnetic fields to shift the VC equilibrium position, this is then utilized to draw a map of the pinning sites in the disk and reconstruct the pinning potential.

The 1 - $\mu$ m-diameter $\mathrm{Ni}_{80} \mathrm{Fe}_{20}(37 \mathrm{~nm}) / \mathrm{Ru}(2 \mathrm{~nm})$ disks is fabricated by electron-beam lithography followed by sputter deposition and lift-off [see scanning electron microscopy (SEM) picture Fig. 1(a)]. A small notch is patterned on one side of the disk to create a small asymmetry in the potential for the VC. $\mathrm{Ti}(10 \mathrm{~nm}) / \mathrm{Au}(110 \mathrm{~nm})$ contacts with a coplanar waveguide geometry are then defined for the high-frequency current injection. X-ray magnetic circular dichroism photoemission microscopy experiments (XMCD-PEEM) ${ }^{16}$ [Fig. 1(b)] reveals a magnetic vortex structure at remanence in agreement with micromagnetic simulations ${ }^{17}$ [Fig. 1(c)].

The resonance of the $\mathrm{VC}$ is detected electrically using a homodyne detection scheme detailed in Ref. 14. The homodyne signal $V_{\mathrm{dc}}$ contains unique information about the dynamics of the $\mathrm{VC}$ and depends directly on the resonance frequency $f_{R}$, the amplitude of oscillation $A(\omega)$ of the $\mathrm{VC}$, and the phase shift $\varphi(\omega)$ between the injected ac current and the oscillating magnetoresistance. It can be expressed as 

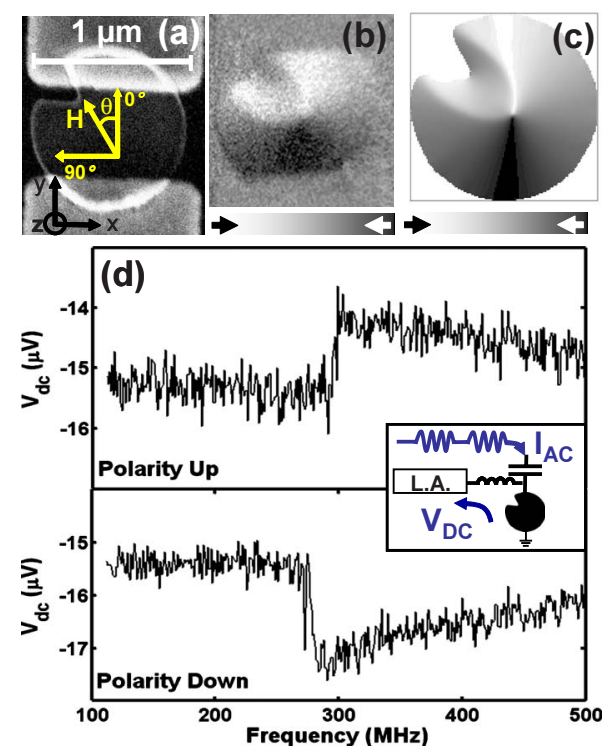

FIG. 1. (Color online) (a) SEM image of a 1- $\mu \mathrm{m}$-diameter disk. The angles used are indicated. (b) XMCD-PEEM image of a $1 \mu \mathrm{m}$ magnetic disk. The gray scale visualizes the horizontal magnetization direction (Ref. 16). (c) Micromagnetic simulation of the magnetic domain structure in a $1-\mu \mathrm{m}$-wide notched magnetic disk. (d) $V_{\text {dc }}$ as a function of the frequency for two different polarities of the VC. Prior to the current injection, the orientation of the VC polarity (up or down) was initialized using a strong $(0.6 \mathrm{~T})$ magnetic field perpendicular to the plane of the magnetic film. Inset: schematic picture of the measurement setup used for the homodyne detection scheme (LA stands for lock-in amplifier). ${ }^{14}$

$$
V_{\mathrm{dc}}(\omega)=\frac{g I_{\mathrm{ac}}}{2} A(\omega) \cos [\varphi(\omega)]
$$

with $g$ the gradient of anisotropic magnetoresistance (AMR) relative to the $\mathrm{VC}$ position.

For the measurement of $V_{\mathrm{dc}}$, a lock-in detection technique was used ${ }^{14}$ [see Fig. 1(d), inset]. Unless specified otherwise, measurements are carried out at room temperature and for an injected power of $-2 \mathrm{dBm}$ corresponding to a current density of $J=1.3 \times 10^{11} \mathrm{~A} / \mathrm{m}^{2}$ (rms). ${ }^{18}$ Figure $1(\mathrm{~d})$ shows the homodyne signal $V_{\mathrm{dc}}$ as a function of the injection current frequency for two VC polarities. A clear resonance is observed for both polarities around $280 \mathrm{MHz}$. In addition, the shape of the homodyne signal depends on the polarity. This can be simply understood from the fact that a $\mathrm{VC}$ with up polarity will gyrate in the opposite sense to a $\mathrm{VC}$ with down polarity. ${ }^{2}$ This leads to opposite phase shifts with respect to the current for the two different polarities resulting in the observed opposite shapes. The polarity of the VC can thus be directly derived from the shape of the homodyne signal.

The dependence of the resonance frequency $f_{R}$ on the VC position in the disk can be studied by applying a small inplane magnetic field that leads to a VC displacement perpendicular to the magnetic field direction. Figure 2(a) shows the dependence of $f_{R}$ on the external magnetic field $H$ applied along the direction $\theta=90^{\circ}$ [see Fig. 1(a)] that illustrates the behavior observed for most angles. We observe: (i) for most field values, a low $f_{R}(\sim 250 \mathrm{MHz})$ and a small dependence
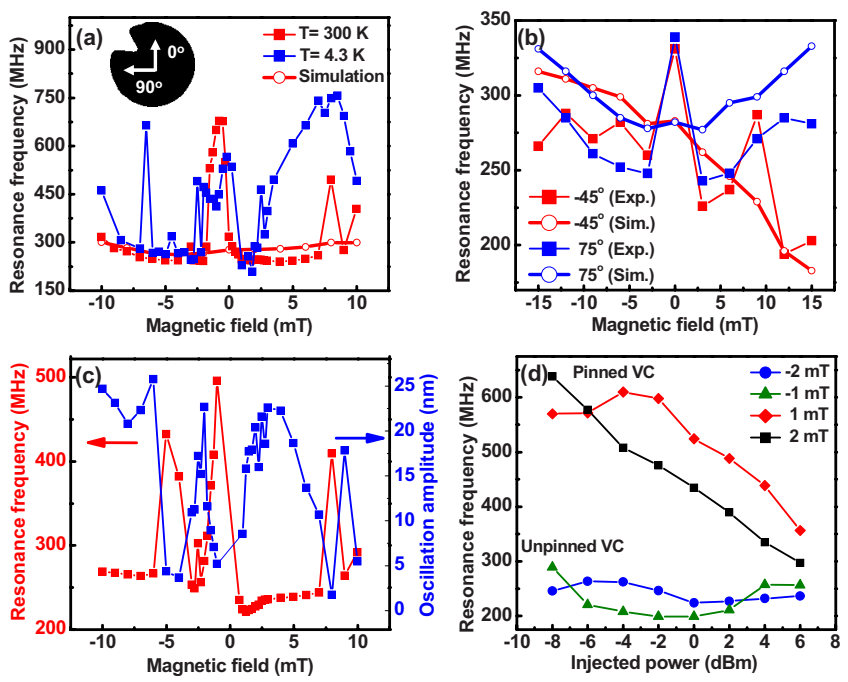

FIG. 2. (Color online) (a) Filled symbols: $f_{R}$ as a function of the magnetic field $H$ applied along $\theta=90^{\circ}$ and for different cryostat temperatures. Open symbols. $f_{R}$ for $\theta=90^{\circ}$ deduced from micromagnetic simulations. (b) Filled symbols: $f_{R}$ as a function of $H$ for $\theta=-45^{\circ}$ and $\theta=75^{\circ}$. Open symbols. Corresponding micromagnetic simulation. (c) $f_{R}$ (red curve) and amplitude of the VC oscillation (blue curve) as a function of $H$ for $\theta=15^{\circ}$. (d) Dependence of $f_{R}$ with the injected power in the case of a pinned VC $(H=1 \mathrm{mT}$ and $H=2 \mathrm{mT})$ and for an unpinned $\mathrm{VC}(H=-1 \mathrm{mT}$ and $H=-2 \mathrm{mT})$ measured at $4.3 \mathrm{~K}$ for $\theta=0^{\circ}$.

on $H$ but additionally (ii) localized peaks with a much higher $f_{R}$. The low and nearly constant $f_{R}$ is actually the behavior expected from the quasiharmonic magnetostatic geometric potential $^{11}$ and can be reproduced well by micromagnetic simulation [see open red symbols in Fig. 2(a)] but the peaks in $f_{R}$ are unexpected. For certain angles such as $\theta=-45^{\circ}$ [Fig. 2(b), red squares], a different behavior is observed with a stronger dependence of the low resonance frequency on $H$ and a clear asymmetry between positive and negative magnetic fields. This contrasts with the more symmetric behavior observed for the other angles as, for example, for $\theta=75^{\circ}$ in Fig. 2(b), blue squares. For $\theta=-45^{\circ}$, the VC is moved toward or away from the notch and thus feels different potentials depending on the field direction, whereas for the other angles, the potential is approximately symmetric. This is confirmed by micromagnetic simulations that reproduce the observed dependence of $f_{R}$ on $H$ (apart from the peaks at around 0 and $9 \mathrm{mT}$ due to pinning sites) [see Fig. 2(b), red and blue empty symbols]. This asymmetric behavior can now be used to detect the chirality of the vortex structure by measuring $f_{R}$ for a small magnetic field along $\theta=-45^{\circ}$ : the VC will move in opposite direction for the two chiralities resulting in a different $f_{R}$.

The appearance of unexpected peaks in $f_{R}$ indicates the presence of local pinning sites of the $\mathrm{VC}$ with large curvature due to the confinement resulting in a local higher resonance frequency. ${ }^{3,19}$ To confirm this picture, we use the amplitude of the homodyne signal $\Delta R$ to deduce the amplitude $A$ of the VC oscillation with $A \sim \Delta R / g$. The AMR gradient $g$ was derived from micromagnetic simulation. A correlation between $f_{R}$ and $A$ is observed from systematic measurements 


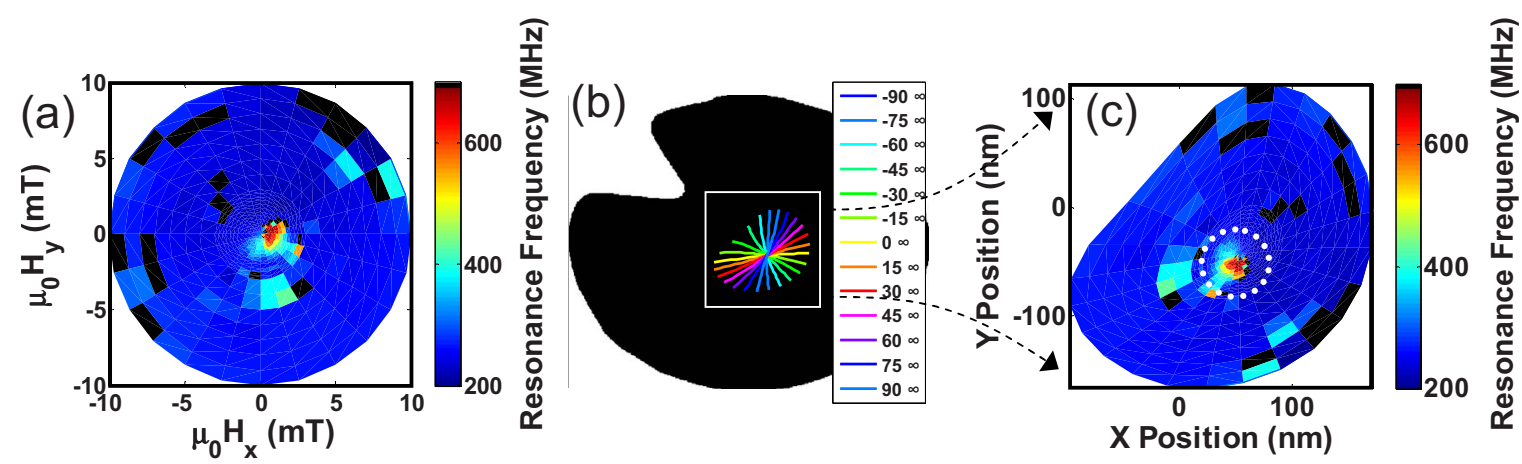

FIG. 3. (Color online) (a) $f_{R}$ as a function of the external in-plane magnetic field $H$ (color coded). Black color shows the absence of a clear resonance in the homodyne signal in the subgigahertz range which also indicates a local pinning of the VC. ${ }^{21}$ (b) Trajectories of the VC for several orientations of the magnetic field with $|H| \leq 10 \mathrm{mT}$ as deduced from micromagnetic simulations. (c) $f_{R}$ plotted in color scale as a function of the VC position.

for different field amplitude $H<10 \mathrm{mT}$ and directions: the gyration radius is on average $7 \mathrm{~nm}$ for $f_{R}>300 \mathrm{MHz}$ (pinned VC) and $25 \mathrm{~nm}$ for $f_{R}<300 \mathrm{MHz}$ (unpinned VC). As an example we present in Fig. 2(c) the dependence of $A$ and $f_{R}$ on the magnetic field, for $\theta=15^{\circ}$ and a clear correlation is observed. This picture is further supported by lowtemperature measurements $(T=4.3 \mathrm{~K})$ where many more magnetic field values exhibit a high $f_{R}$ when sweeping $H$ [see Fig. 2(a), blue curve]. The lower thermal excitation allows the pinning sites with lower pinning energy to trap the $\mathrm{VC}$ so that it is effectively pinned by much more pinning sites. Additional information about the local pinning potential can be obtained from the power dependence of $f_{R}$ [Fig. $2(\mathrm{~d})]$. For the case of the unpinned $\mathrm{VC}$ with low $f_{R}$ $(\sim 250 \mathrm{MHz})$, a small dependence of $f_{R}$ is observed in agreement with a quasiharmonic potential to first order. For the case of the pinned VC with high $f_{R}$, a strong decrease in $f_{R}$ with the injected power is observed revealing the nonharmonicity of the pinning potential. ${ }^{20}$

The strong increase in $f_{R}$ induced by local pinning of the $\mathrm{VC}$ can be used to draw a map of the pinning sites in the magnetic disk. This is done by first carrying out a systematic measurement of $f_{R}$ as a function of the in-plane magnetic field amplitude and direction [see Fig. 3(a)]. Using micromagnetic simulations, we then deduce for each applied inplane magnetic field the position of the $\mathrm{VC}$ in the disk. ${ }^{23}$ Figure 3(b) shows the resulting trajectories of the $\mathrm{VC}$ for different magnetic field directions. A spatial map of $f_{R}$ with the VC position can now be drawn and hence the position and relative strength of the pinning sites are shown [Fig. 3(c)]. One can see that the pinning sites seem to be randomly distributed and highly localized in the disk with an average size on the order of $\sim 20 \mathrm{~nm}$. Contrary to previous results, ${ }^{3}$ the pinning sites are not due to the general grain structure of the film in our case but more likely to random local defects in the film. This can be explained from the large amplitude of oscillation for the unpinned case, which is of the order or larger than the mean grain size of our films as deduced from atomic force microscopy (AFM) measurement $(\approx 30 \mathrm{~nm})$ so that only the strongest pinning sites here play a role for our room-temperature measurements. This spatial map of the resonance frequency can be used to reconstruct the local magnetic potential in the film by numerical integra- tion of the curve $f_{R}(\mathbf{X})$. As an example, Fig. 4(a) shows a three-dimensional (3D) plot of the magnetic potential as a function of the VC position of a strong pinning site localized close to the center of the disk. Due to the local pinning, the potential is strongly curved compared to the potential expected from the geometrical pinning, as can be seen in Fig. 4(b). Whereas the geometrical potential is well fitted by a parabolic potential for the considered VC displacement range, the pinning potential is markedly nonharmonic and can only be fitted by a fourth-order polynomial. This can be visualized in Fig. 4(b) by comparing the pinning potential (blue points) to its parabolic part obtained from the curvature at the bottom of the pinning potential (black line). Similar to the potential obtained by Bedau et al. ${ }^{20}$ for a vortex wall pinned in a notch, the measured potential lies below its harmonic part and thus flattens off away from the origin.

In addition to the resonance frequency and the oscillation amplitude, the homodyne signal contains unique information about the phase $\varphi(\omega)$, which has not been previously used. We find from systematic measurements that the shape of the dc signal strongly depends on the position of the VC. As an
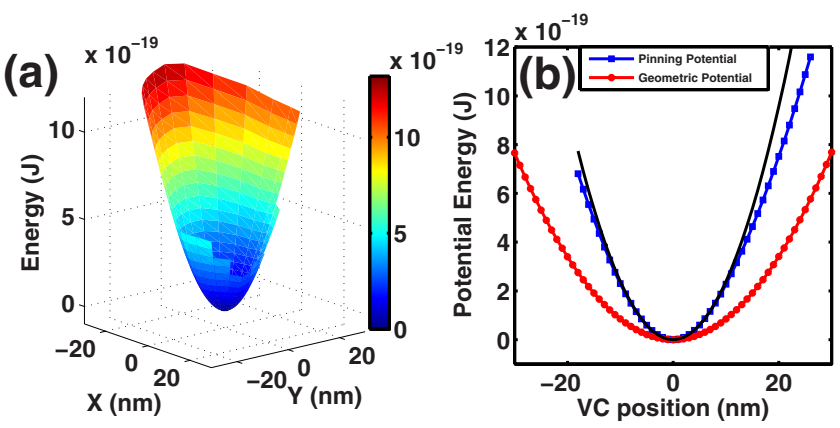

FIG. 4. (Color online) (a) Pinning potential of the pinning site marked with a white circle in Fig. 3(c), obtained by numerical integration of the curves $f_{R}(H)$. The VC position is calculated from the center of the pinning site. (b) Blue curve: cut along the direction $\theta=0$ of the pinning potential. Red curve: geometrical potential calculated from the center of the disk and obtained by numerical integration of the baseline of $f_{R}(H)$ in Fig. 2(a). Black line: harmonic part of the pinning potential as a function of the VC position, obtained from the curvature of the pinning potential at the bottom of the well. 
example, we show in Figs. 5(a)-5(c) three $V_{\mathrm{dc}}(f)$ curves characterized by different shapes and measured at the same magnetic field amplitude $(H=+6 \mathrm{mT})$, but at different angles $\left(\theta=-150^{\circ}, \theta=-75^{\circ}\right.$, and $\theta=0^{\circ}$, respectively). These different shapes indicate a strong variation in the phase shift $\varphi$ with the position of the VC in the disk. To further quantify these observations, we consider a simple one-dimensional model where the dynamics of the $\mathrm{VC}$ is described by a modified Thiele equation with the force $\mathbf{F}_{\mathbf{I}}$ due to the ac current excitation included, ${ }^{10,18}$

$$
\mathbf{G} \times \dot{\mathbf{X}}+\mathbf{F}_{\mathbf{m}}+\mathbf{F}_{\mathbf{I}}-\alpha D \dot{\mathbf{X}}=0 .
$$

Here $\mathbf{F}_{\mathbf{m}}$ is the force due to the magnetostatic potential well and the external static magnetic field, $\mathbf{X}$ is the VC position, $\mathbf{G}=-G_{0} p \mathbf{e}_{\mathrm{z}}$ is the gyrovector with $p= \pm 1$ the polarity, $\alpha$ is the Gilbert damping, and $D$ the damping tensor. ${ }^{10}$ The force $\mathbf{F}_{\mathbf{I}}$ has two origins: ${ }^{18}$ (i) the force due to the spin torque $\mathbf{F}_{\mathbf{S T}}=-\mathbf{G} \times \mathbf{u}+\beta D \mathbf{u}$ with $\mathbf{u}=\mu_{B} J P / e M_{s} \mathbf{e}_{\mathbf{y}}\left(M_{s}\right.$ is the saturation magnetization, $P$ the spin polarization, and $J$ the current density) and $\beta$ the nonadiabatic factor; (ii) the force $\mathbf{F}_{\mathbf{O e}}$ due to the Oersted field along the $x$ axis. This force can be approximated as $\mathbf{F}_{\mathbf{O e}}=-c \gamma G_{0} u \mathbf{e}_{\mathbf{y}}$ with $c= \pm 1$ the chirality and $\gamma$ the ratio between the Oersted field force and the adiabatic spin-transfer force. ${ }^{18}$ One can use this dynamical equation to derive $^{15,18}$ an analytical expression for the amplitude of oscillation $A(\omega)$ and the phase shift $\varphi(\omega)$. Assuming a local harmonic and symmetric potential well, $\varphi(\omega)$ can be written in a simple form: $\varphi(\omega)=\eta_{0}+\epsilon+\delta(\omega)$ with $\eta_{0}$ the angle between the local AMR gradient and the direction of the electron flow, $\epsilon=\arctan \left[p\left(\gamma c-\beta_{0}\right)\right]$ and $\delta(\omega)$ $=\arctan \left[p\left(\omega_{R}^{2}-\omega^{2}\right) / 2 \alpha_{0} \omega^{2}\right]$ with $\beta_{0}=\beta D / G_{0}, \alpha_{0}=\alpha D / G_{0}$, and $\omega_{R}=2 \pi f_{R}$ (see Appendix).

By fitting our experimental data using Eq. (1) and the analytical expressions of $A(\omega)$ and $\varphi(\omega)$, the relative phase shift $\eta=\eta_{0}+\epsilon$ and $\omega_{R}$ can be extracted from the $V_{\mathrm{dc}}(f)$ measurements. Examples of fits are shown as red lines in Figs. $5(\mathrm{a})-5(\mathrm{c})$. As expected, the three curves $V_{\mathrm{dc}}(f)$ in Figs. 5(a)-5(c) are characterized by different phase shifts $\eta=2.5$, $\eta=1.65$, and $\eta=0.45$, respectively, which account for their different shapes. More information can be obtained from the map of the phase shift $\eta$ as a function of the magnetic field that can be obtained from a systematic fitting of our experimental results. As can be seen in Fig. 5(d), $\eta$ continuously varies from 0 to $2 \pi$ as the magnetic field is rotated which illustrates the local change in the AMR gradient orientation $\eta_{0}$ with the position of the VC in the disk. To confirm this picture, we calculated $\eta_{0}$ from micromagnetic simulations for different magnetic field directions and amplitudes. As can be seen in Fig. 5(e), the resulting map is similar to the experimental phase shift but is rotated about $75^{\circ}$ clockwise. Such a shift indicates an additional phase shift of $\epsilon=\arctan \left[p\left(\gamma c-\beta_{0}\right)\right]$ due to the contribution of the Oersted field and the nonadiabatic torque. By fitting the simulated distribution of $\eta$ with the experimental one, a phase shift $\epsilon=1.26 \pm 0.05$ results leading to $\gamma c-\beta_{0}=3.1 \pm 0.5$. The parameter $\beta$ is found to be of the order or a few times larger ${ }^{22}$ than the damping constant $\alpha \approx 0.01$ in $\mathrm{Ni}_{80} \mathrm{Fe}_{20}$ so $\beta_{0}$ $\sim 0.03$ with $D / G_{0} \sim 3.3$ and thus the phase shift is primarily
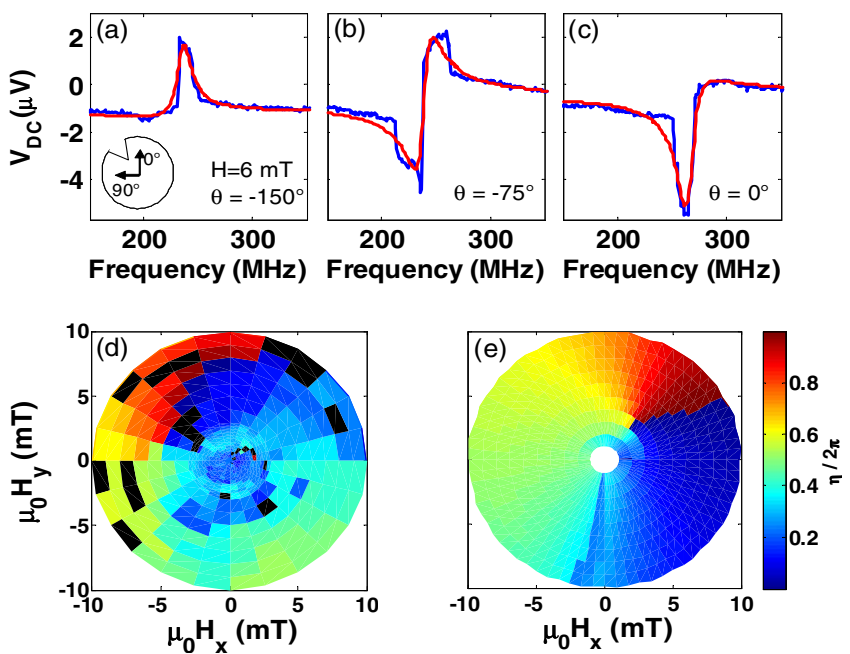

FIG. 5. (Color online) $[(\mathrm{a})-(\mathrm{c})]$ Blue lines: $V_{\mathrm{dc}}$ as a function of the frequency of the injected current for $H=6 \mathrm{mT}$ and (a) $\theta=-150^{\circ}$, (b) $\theta=-75^{\circ}$, and (c) $\theta=0^{\circ}$. Red lines: from fitting using Eq. (1), we determined the different phase shifts (a) $\eta=2.51$, (b) $\eta=1.65$, and (c) $\eta=0.45$. (d) Experimental phase shift $\eta$ in color scale as function of the in-plane magnetic field. (e) Simulated phase shift $\eta_{0}$ due to the angle between the direction of AMR gradient and the current in color scale showing a $75^{\circ}$ rotation compared to the experimental phase shift.

due to the effect of the Oersted field. Using a value of spin polarization of $P=0.7$ in $\mathrm{Py},{ }^{4}$ this leads to an estimation of the Oersted field of about $4.3 \pm 1$ Oe rms for $J=1.3$ $\times 10^{11} \mathrm{~A} / \mathrm{m}^{2}$. Our analysis of the phase shift distribution thus allows us to clearly separate the contribution of the spin torque and the Oersted field effect, which accounts in our experiments for up to $75 \%$ of the force exerted on the VC. This conclusion is supported by $3 \mathrm{D}$ electromagnetic simulations we carried out for our specific geometry. As expected, the amplitude of the transverse component of the Oersted field varies rapidly in the thickness of the magnetic disk with, respectively, 10 Oe and -3.4 Oe at the top and bottom edges of the disk for $J=1.3 \times 10^{11} \mathrm{~A} / \mathrm{m}^{2}$. This means that there is a general offset of around 3.3 Oe of the Oersted field as compared to a fully homogeneous current distribution in the disk. The simulations show that this Oersted field originates from the vertical currents at the permalloy/gold interface due to the geometry of the contact and the difference in conductivity, as well as an inhomogeneous current distribution in the disk.

In conclusion, the resonant excitation of a magnetic vortex core in a magnetic disk by a high-frequency current is characterized. A fast electrical detection of the resonance is obtained by using the rectification of the ac current induced by the oscillating vortex core (homodyne technique). We show that in our asymmetric disk, the polarity and the chirality of the vortex state can be determined from the homodyne rectification signal, which also yields the amplitude and the phase shift of the oscillating VC. The analysis of the phase shift distribution in the disk allows a clear discrimination between the contribution of the adiabatic spin torque and the Oersted field which is the main force driving the VC dynamics in our disk. The dominating Oersted field can explain the 
earlier observation that the current induced VC switching occurs at current densities far below the threshold current density predicted for pure spin torque induced reversal. ${ }^{20}$ Our measurements show furthermore that local pinning sites in the disk lead to a distinct signature in the homodyne signal with increased resonance frequency and this is used to obtain a spatial map of the pinning sites in the disk. The observed strong influence of the local pinning sites on the VC dynamics may play an important role for any device based on the manipulation of nanoscale confined magnetic object such as a VC or domain wall. Our efficient method of characterization in conjunction with structural investigations such as high-resolution electron microscopy may open a path to the identification of the defect types and resulting materials improvement for devices with reliable dynamical switching.

\section{ACKNOWLEDGMENTS}

This work is supported by the EU-Research Training Network SPINSWITCH (Contract No. MRTN-CT-2006035327), the Samsung Advanced Institute of Technology, the DFG (SFB 767, Grant No. KL 1811), the Swiss National Science Foundation, the Landesstiftung Baden Württemberg, and the European Research Council via a Starting Independent Researcher Grant (Grant No. ERC-2007-Stg 208162).

\section{APPENDIX}

\section{Analytical expression of the dc voltage}

The dynamics of the vortex core is described by a modified Thiele equation where the force $\mathbf{F}_{\mathbf{I}}$ due to the ac current excitation is included, ${ }^{10,24-26}$

$$
\mathbf{G} \times \dot{\mathbf{X}}+\mathbf{F}_{\mathbf{m}}+\mathbf{F}_{\mathbf{I}}-\alpha D \dot{\mathbf{X}}=0 .
$$

Here $\mathbf{F}_{\mathbf{m}}$ is the force due to the magnetostatic potential well and the external static magnetic field, $\mathbf{X}$ is the vortex core position, and $\mathbf{G}=G_{0} p \mathbf{e}_{\mathbf{z}}$ is the gyrovector with $G_{0}=2 \pi t M_{S} / \gamma_{G}\left(M_{s}\right.$ is the saturation magnetization, $\gamma_{G}$ the gyromagnetic factor, $t$ the thickness of the disk, $p= \pm 1$ the polarity, $\alpha$ the Gilbert damping, and $D$ the damping tensor). ${ }^{10}$ The current-induced force $\mathbf{F}_{\mathbf{I}}$ is the sum of two terms: (i) the force due to the spin torque ${ }^{24,25} \mathbf{F}_{\mathbf{S T}}=-\mathbf{G} \times \mathbf{u}$ $+\beta D \mathbf{u}$ with $\mathbf{u}=\mu_{B} J P / e M_{s} \mathbf{e}_{\mathbf{y}}(P$ is the spin polarization and $J$ the current density) and $\beta$ the nonadiabatic factor; (ii) the force $\mathbf{F}_{\mathbf{O e}}=-\frac{\partial W_{Z}}{\partial \mathbf{X}}$ due to the Oersted field with $W_{Z}$ the Zeeman energy associated with the Oersted field $\mathbf{H}_{\mathbf{O e}}$. If we assume an homogeneous Oersted field $H_{\mathrm{Oe}}$ along the $x$ direction [see Fig. 1(a)], $W_{Z}$ can be approximated for small VC displacement from the disk center $Y \mathbf{e}_{\mathbf{Y}}$ by $W_{Z} \approx-c \mu_{0} M_{s} \pi R t H_{\mathrm{Oe}} Y$ with $c= \pm 1$ the chirality, $R$ the radius of the disk, and $t$ the thickness of the magnetic film. ${ }^{27}$ The force $\mathbf{F}_{\mathbf{O e}}$ can thus be written as $\mathbf{F}_{\mathbf{O e}}=c \mu_{0} M_{s} \pi R t H_{\mathrm{Oe}} \mathbf{e}_{\mathbf{y}}$. As $H_{\mathrm{Oe}}$ is proportional to the current density, we can write $\mathbf{F}_{\mathbf{O e}}=-c \gamma G_{0} u \mathbf{e}_{\mathbf{y}}$ with $\gamma=-\mu_{0} M_{s} \pi R t H_{\mathrm{Oe}} / G_{0} u$ a dimensionless parameter that describes the amplitude of the Oersted field force relative to the adiabatic spin-transfer force. In the following, we assume that the local potential well $W_{m}$ due to the magnetostatic, exchange and Zeeman energy is harmonic and with radial symmetry $^{11,27}$ and can be expressed as $W_{m}=\frac{1}{2} k r^{2}$ with $r$ the distance of the vortex core from its equilibrium position $\mathbf{X}_{\mathbf{0}}$ for a given external magnetic field and $k$ the local stiffness of the potential. $\mathbf{F}_{\mathbf{m}}$ thus reads as $\mathbf{F}_{\mathbf{m}}=-\frac{\partial W_{m}}{\partial X}=-k \mathbf{r}$ with $\mathbf{r}=\mathbf{X}-\mathbf{X}_{\mathbf{0}}$.

By projecting Eq. (A1) on the $x$ and $y$ axes, $V_{\mathrm{dc}}$ can be written for a small amplitude of oscillation and an exciting current $I(t)=I_{\mathrm{ac}} \cos (\omega t),{ }^{15}$

$$
V_{\mathrm{dc}}=\frac{I_{\mathrm{ac}} g}{2}\left[\cos \left(\eta_{0}\right) Y(\omega)-p X(\omega) \sin \left(\eta_{0}\right)\right] .
$$

$g=|\partial \Delta R / \partial \mathbf{X}|$ is the gradient of AMR relative to the VC position $\mathbf{X}$ and $\eta_{0}$ the angle between $\mathbf{e}_{\mathbf{y}}$ and the direction of the AMR gradient $\mathbf{g}$. The amplitude of oscillation in the $(x, y)$ plane $X(\omega)$ and $Y(\omega)$ can be expressed as ${ }^{15}$

$$
X(\omega)=-C_{\omega}\left[-\left(\omega_{R}^{2}-\omega^{2}\right) q+\alpha^{*} \omega^{2}\left(\beta_{0}-c \gamma-\alpha_{0}\right)\right]
$$

$$
Y(\omega)=C_{\omega}\left\{\left(\omega_{R}^{2}-\omega^{2}\right)\left(\beta_{0}-c \gamma\right) q+\alpha^{*} \omega^{2}\left[\alpha_{0}\left(\beta_{0}-c \gamma\right)+1\right]\right\}
$$

with

$$
C_{\omega}=\frac{u_{0}}{\left(1+\alpha_{0}^{2}\right)\left[\left(\omega_{R}^{2}-\omega^{2}\right)^{2}+\left(\alpha^{*} \omega\right)^{2}\right]}
$$

and

$$
\begin{gathered}
\alpha_{0}=\frac{\alpha D}{G_{0}}, \quad \beta_{0}=\frac{\beta D}{G_{0}}, \quad q=\frac{k D}{G_{0}}, \\
\omega_{R}=\frac{q}{\sqrt{1+\alpha_{0}^{2}}}, \quad \alpha^{*}=\frac{2 q \alpha_{0}}{1+\alpha_{0}^{2}} .
\end{gathered}
$$

Equation (A2) can be written in the form,

$$
V_{\mathrm{dc}}=\frac{g I_{\mathrm{ac}}}{2} A(\omega) \cos [\varphi(\omega)]
$$

with $A(\omega)=\sqrt{\left(X^{2}+Y^{2}\right)}$ and $\varphi(\omega)=\eta_{0}+\delta(\omega)$ with $\delta(\omega)$ $=\arctan (p X / Y)$.

One can write $\delta(\omega)$ as

$$
\begin{gathered}
\delta=\frac{\pi}{2}+\arctan (-p Y / X) \\
=\frac{\pi}{2}-p \arctan \left[\frac{\beta_{0}-c \gamma+\alpha^{*} \omega^{2} \frac{\alpha_{0}\left(\beta_{0}-c \gamma\right)+1}{\left(\omega_{R}^{2}-\omega^{2}\right) q}}{1-\frac{\alpha_{0} \omega^{2}\left(\beta_{0}-c \gamma-\alpha_{0}\right)}{\left(\omega_{R}^{2}-\omega^{2}\right) q}}\right] .
\end{gathered}
$$

For $\gamma \gg \beta_{0}, \alpha_{0}$, this expression can be simplified as

$$
\varphi(\omega) \approx \eta_{0}+\epsilon+\delta(\omega)
$$

with

$$
\epsilon=\arctan \left[-p\left(\beta_{0}-c \gamma\right)\right]
$$




$$
\delta(\omega)=\arctan \left[\frac{p q}{\alpha^{*}} \frac{\left(\omega_{R}^{2}-\omega^{2}\right)}{\omega^{2}}\right] .
$$

We used Eq. (A6) in combination with Eq. (A9) to fit our data.

For the fitting procedure, we use the following relation for the damping tensor: ${ }^{15,28,29}$

$$
\frac{D}{G_{0}}=1+\frac{1}{2} \ln \left(\frac{R}{\delta_{R}}\right)
$$

with $R=500 \mathrm{~nm}$ the radius of the disk and $\delta_{R} \approx 5 \mathrm{~nm}$ the radius of the vortex core. We use in addition $\alpha=0.01$, $P=0.7$ for permalloy ${ }^{30}$ and assume $\beta=2 \alpha$. For the fitting, $g, \eta=\eta_{0}+\epsilon$ and $\omega_{R}$ were used as free parameters.

\section{Micromagnetic simulation}

Micromagnetic simulations were performed using the software OOMMF. ${ }^{17}$ The shape of the disk used for the simulation was drawn from a SEM image of the disk. The resonance frequency of the gyrotropic mode for each value of the magnetic field was obtained by first moving slightly the vortex core out of its equilibrium position and then letting it relax toward equilibrium. The resonance frequency was deduced from the Fourier transformation of the magnetization during the relaxation process. Standard magnetic parameters of permalloy were used for the simulation with $M_{s}=800 \times 10^{3} \mathrm{~A} / \mathrm{m}$ and $A=13 \times 10^{-12} \mathrm{~J} / \mathrm{m}$.

\section{Additional informations on the experimental setup}

The homodyne signal was measured using a lock-in detection technique: the high-frequency current is modulated with a $7 \mathrm{kHz}$ square wave and injected into the highfrequency part of the bias tee [see Fig. 1(d), inset]; $V_{\mathrm{dc}}$ is measured with a lock-in amplifier connected to the dc part of the bias tee and phase locked to the modulation signal. The current density was deduced from the resistance increase induced by the Joule heating when injecting the microwave power. We corrected for the frequency-dependent power reflection and losses so that the current density in the structure is constant for all frequencies.
*Also at the Laboratory for Micro- and Nanotechnology, Paul Scherrer Institut, CH-5232 Villigen PSI, Switzerland.

${ }^{\dagger}$ Also at Laboratory of Nanomagnetism and Spin Dynamics, Ecole Polytechnique Fédérale de Lausanne (EPFL), 1015 Lausanne, Switzerland; SwissFEL, Paul Scherrer Insititut, 5232 Villigen PSI, Switzerland; mathias.klaeui@magnetism.ch

${ }^{1}$ T. Shinjo, T. Okuno, R. Hassdorf, K. Shigeto, and T. Ono, Science 289, 930 (2000).

${ }^{2}$ S.-B. Choe, Y. Acremann, A. Scholl, A. Bauer, A. Doran, J. Stöhr, and H. A. Padmore, Science 304, 420 (2004).

${ }^{3}$ R. L. Compton and P. A. Crowell, Phys. Rev. Lett. 97, 137202 (2006).

${ }^{4}$ S. Kasai, Y. Nakatani, K. Kobayashi, H. Kohno, and T. Ono, Phys. Rev. Lett. 97, 107204 (2006).

${ }^{5}$ B. Van Waeyenberge, A. Puzic, H. Stoll, K. W. Chou, T. Tyliszczak, R. Hertel, M. Fähnle, H. Brückl, K. Rott, G. Reiss, I. Neudecker, D. Weiss, C. H. Back, and G. Schütz, Nature (London) 444, 461 (2006).

${ }^{6}$ K. Yamada, S. Kasai, Y. Nakatani, K. Kobayashi, H. Kohno, A. Thiaville, and T. Ono, Nature Mater. 6, 269 (2007).

${ }^{7}$ M. Bolte, G. Meier, B. Krüger, A. Drews, R. Eiselt, L. Bocklage, S. Bohlens, T. Tyliszczak, A. Vansteenkiste, B. Van Waeyenberge, K. W. Chou, A. Puzic, and H. Stoll, Phys. Rev. Lett. 100, 176601 (2008).

${ }^{8}$ M. Curcic, B. Van Waeyenberge, A. Vansteenkiste, M. Weigand, V. Sackmann, H. Stoll, M. Fähnle, T. Tyliszczak, G. Woltersdorf, C. H. Back, and G. Schütz, Phys. Rev. Lett. 101, 197204 (2008).

${ }^{9}$ A. Wachowiak, J. Wiebe, M. Bode, O. Pietzsch, M. Morgenstern, and R. Wiesendanger, Science 298, 577 (2002).

${ }^{10}$ A. A. Thiele, Phys. Rev. Lett. 30, 230 (1973).

${ }^{11}$ K. Y. Guslienko, B. A. Ivanov, V. Novosad, Y. Otani, H. Shima, and K. Fukamichi, J. Appl. Phys. 91, 8037 (2002).
${ }^{12}$ S. Bohlens, B. Krüger, A. Drews, M. B. G. Meier, and D. Pfannkuche, Appl. Phys. Lett. 93, 142508 (2008).

${ }^{13}$ S. Kasai, P. Fischer, M.-Y. Im, K. Yamada, Y. Nakatani, K. Kobayashi, H. Kohno, and T. Ono, Phys. Rev. Lett. 101, 237203 (2008).

${ }^{14}$ D. Bedau, M. Kläui, S. Krzyk, U. Rüdiger, G. Faini, and L. Vila, Phys. Rev. Lett. 99, 146601 (2007).

${ }^{15}$ R. Moriya, L. Thomas, M. Hayashi, Y. B. Bazaliy, C. Rettner, and S. S. P. Parkin, Nat. Phys. 4, 368 (2008).

${ }^{16}$ B. Hermsmeier, J. Stöhr, Y. Wu, M. Samant, G. Harp, S. Koranda, D. Dunham, and B. P. Tonner, J. Appl. Phys. 75, 6890 (1994).

${ }^{17}$ M. Donahue and D. Porter, oOMmF User's Guide, V 1.1b, 2004.

${ }^{18}$ See Appendix.

${ }^{19}$ R. L. Compton, T. Y. Chen, and P. A. Crowell, Phys. Rev. B 81, 144412 (2010)

${ }^{20}$ D. Bedau, M. Kläui, M. T. Hua, S. Krzyk, U. Rüdiger, G. Faini, and L. Vila, Phys. Rev. Lett. 101, 256602 (2008).

${ }^{21}$ K. Kuepper, L. Bischoff, Ch. Akhmadaliev, J. Fassbender, H. Stoll, K. W. Chou, A. Puzic, K. Fauth, D. Dolgos, G. Schütz, B. Van Waeyenberge, T. Tyliszczak, I. Neudecker, G. Woltersdorf, and C. H. Back, Appl. Phys. Lett. 90, 062506 (2007).

${ }^{22}$ M. Eltschka, M. Wötzel, J. Rhensius, S. Krzyk, U. Nowak, M. Kläui, T. Kasama, R. E. Dunin-Borkowski, L. J. Heyderman, H. J. van Driel, and R. A. Duine, Phys. Rev. Lett. 105, 056601 (2010).

${ }^{23}$ To confirm the position, the AMR was also simulated for each $\mathrm{VC}$ position and found to be consistent with the one measured experimentally.

${ }^{24}$ A. Thiaville, Y. Nakatani, J. Miltat, and Y. Suzuki, Europhys. Lett. 69, 990 (2005).

${ }^{25}$ J. Shibata, Y. Nakatani, G. Tatara, H. Kohno, and Y. Otani, Phys. Rev. B 73, 020403 (2006). 
${ }^{26}$ B. Krüger, D. Pfannkuche, M. Bolte, G. Meier, and U. Merkt, Phys. Rev. B 75, 054421 (2007).

${ }^{27}$ K. Y. Guslienko, V. Novosad, Y. Otani, H. Shima, and K. Fukamichi, Appl. Phys. Lett. 78, 3848 (2001).
${ }^{28}$ K. Y. Guslienko, Appl. Phys. Lett. 89, 022510 (2006).

${ }^{29}$ K. Y. Guslienko and K. L. Metlov, Phys. Rev. B 63, 100403 (2001).

${ }^{30}$ J. Bass and W. P. Pratt, J. Magn. Magn. Mater. 200, 274 (1999). 